\title{
Characterisation of natural killer cells and CD56+ T-cells in sarcoidosis patients
}

\author{
K. Katchar*, K. Söderström*, J. Wahlstrom*, A. Eklund* and J. Grunewald*
}

ABSTRACT: The aim of the current study was to investigate the frequency, phenotype and functional activity of natural killer (NK) cells and CD56+ T-cells in the bronchoalveolar lavage fluid and peripheral blood of patients with pulmonary sarcoidosis when compared with healthy volunteers, using staining with a panel of monoclonal antibodies followed by flow cytometry.

The results revealed that the majority of the lung NK cell subpopulation expressed CD56bright. In contrast, there was a predominant CD56dim subset in the blood of both patients and healthy controls. Most lung NK cells expressed C-type lectin-like human leukocyte antigen (HLA)-Especific inhibitory receptor (i.e. CD94/NKG2A), but only a few lung NK cells expressed killer cell immunoglobulin-like inhibitory receptors specific for HLA-A, -B or -C molecules. In addition, a significantly increased number of CD56+ T-cells were observed in the blood of patients when compared with controls. Upon in vitro stimulation, both lung NK and CD56+ T-cells produced considerable amounts of interferon- $\gamma$ and tumour necrosis factor- $\alpha$.

Thus, in the lungs of patients with pulmonary sarcoidosis, a distinct phenotype of natural killer cells with the capacity to produce cytokines and actively participate in the T-helper 1-like inflammatory response associated with sarcoidosis was identified.

KEYWORDS: Bronchoalveolar lavage fluid, CD56+ T-cells, killer inhibitory receptors, natural killer cells, sarcoidosis

A s well as having a cytotoxic function, natural killer (NK) cells have the capacity to secrete a broad panel of pro- and antiinflammatory cytokines. Through these effector functions, they are known to contribute to the defence against many pathogens and tumours [1]. According to the expression density of CD56, human NK cells in the peripheral blood (PB) can be broadly divided into two major subgroups: the CD56bright and CD56dim subsets. The minor CD56bright subset is capable of producing substantial levels of inflammatory cytokines (e.g. tumour necrosis factor (TNF)- $\alpha$, interferon (IFN)$\gamma)$ and may have a particularly important role in immune regulation. The major CD56dim subset shows marked cytotoxicity, but is less prone to producing cytokines [2, 3].

In order to distinguish normal cells from stressed or aberrant cells, NK cells express a variety of activating and inhibitory receptors that regulate their cytotoxicity and cytokine release. The inhibitory receptors mainly focus on major histocompatibility complex (MHC) class I molecules and fall into different families. The killer cell immunoglobulin-like receptors (KIRs) or leukocyte immunoglobulin-like receptors primarily recognise human leukocyte antigen (HLA)-A,
-B, -C or - $\mathrm{G}$ molecules, whereas the C-type lectinlike receptor dimer CD94/NKG2A binds HLA-E when loaded with HLA class I-derived leader peptides [4].

Activated T-cells can express a wide variety of receptors commonly found on NK cells, including CD56, KIRs and CD94/NKG2A receptors, of which some apparently associate with distinct effector functions. For example, the CD56 marker expressed on PB T-cells closely correlates with cytolytic effector T-cells, which can mediate nonMHC-restricted cytotoxicity [5, 6]. Most of these CD56+ T-cells express CD8 and appear more efficient in IFN- $\gamma$ production, as compared with CD56- T-cells [7].

Sarcoidosis is a granulomatous disease of unknown cause, which frequently affects intrathoracic organs, most commonly the lungs. Previous studies investigating NK cell distribution and function, mainly using immunohistological techniques, has indicated an altered NK cell cytotoxic activity in the lungs of patients with sarcoidosis [8-10]. In the present study, the frequency of NK and CD56+ T-cells in bronchoalveolar lavage fluid (BALF) and paired $\mathrm{PB}$ samples from sarcoidosis patients and healthy
AFFILIATIONS

*Dept of Medicine, Division of Respiratory Medicine, Karolinska Hospital, Stockholm, Sweden \#Dept of Pathology, Stanford Medical School, Stanford, CA, USA.

CORRESPONDENCE

K. Katchar

Beth Israel Deaconess Medical Center

Dana 601

330 Brookline Avenue

Boston

MA 02215

USA

Fax: 16176678144

E-mail: kkatchar@bidmc.harvard.edu

Received:

March 152005

Accepted after revision:

April 012005

SUPPORT STATEMENT

The authors gratefully acknowledge grant support from Swedish Heart Lung Foundation, The Swedish Medical Society, The Swedish Medical Research Council (Grant521-2003-4733) and Karolinska Institutet (Stockholm, Sweden). 
controls was determined. The percentage of CD56bright (cytokine-producing) and CD56dim (cytotoxic) NK cells in lung and blood cells from patients and controls was analysed. In addition, the expression of KIRs and CD94/NKG2A receptors on paired BALF and PB NK and CD56+ T-cells in both patients and controls was determined. Finally, the cytokine production (IFN- $\gamma$ and TNF- $\alpha$ ) capacity of these cell populations after in vitro stimulation was examined.

\section{MATERIALS AND METHODS Subjects}

Paired samples of BALF cells and PB cells were obtained from a total of 19 Scandinavian sarcoidosis patients (mean age 40 yrs, range 26-57). All patients had a clinical presentation and chest radiographic findings that were typical for pulmonary sarcoidosis. All patients were classified as having an ongoing active disease, as assessed on the basis of symptoms, chest radiography and pulmonary function tests, using previously established criteria [11]. The radiographic stages and pulmonary function tests are indicated in table 1. Eight of the patients were female and 11 were male. One of the subjects was a smoker, three were ex-smokers and 15 had never smoked. Sixteen patients had biopsy-proven sarcoidosis and the three remaining patients had either classical Löfgren's syndrome or a BAL CD4/CD8 ratio $>4.0$, thus strongly supporting the diagnosis [12]. None of the patients was undergoing any treatment prior to the BAL procedure. Disease duration was defined as the time from onset of symptoms to BAL procedure.

A total of 20 healthy volunteers were also included in the study. There were 14 females and six males, with mean (range) age 30 yrs (22-53), all nonsmoking, without signs of respiratory tract infection and normal chest radiography (table 1). All subjects gave their informed consent and the study was approved by the local ethics committee.

\begin{tabular}{|c|c|c|c|}
\hline \multirow{2}{*}{$\begin{array}{l}\text { TABLE } 1 \\
\text { Characterist }\end{array}$} & \multicolumn{3}{|c|}{$\begin{array}{l}\text { Bronchoalveolar lavage fluid (BALF) cell } \\
\text { characteristics and clinical information from } \\
\text { sarcoidosis patients }{ }^{\#} \text { and healthy controls }\end{array}$} \\
\hline & & Patients & Controls \\
\hline Total BALF & IIs $\times 10^{6} \cdot \mathrm{L}^{-1}$ & $153.0(84.6-400.0)$ & $73.5(49.8-112.9)^{+}$ \\
\hline \multicolumn{4}{|c|}{ Cell types $\%$ of total BALF } \\
\hline Alveolar $\mathrm{m}$ & crophages & $83.0(21.0-96.0)$ & $93.6(82.6-97.4)$ \\
\hline Lymphocy & & $16.0(3.2-78.8)$ & $5.2(2.0-16.0)$ \\
\hline Neutrophi & & $0.6(0.2-5.0)$ & $1.0(0.0-4.5)$ \\
\hline Eosinophi & & $0.0(0.0-1.2)$ & $0.0(0.0-4.0)$ \\
\hline BALF CD4/ & ว cell ratio & $4.4(0.7-12.6)$ & $2.1(0.7-6.8)$ \\
\hline \multicolumn{4}{|c|}{ Lung function parameters ${ }^{\S}$} \\
\hline VC \% pres & & $102(72-121)$ & ND \\
\hline$D \mathrm{~L}, \mathrm{CO} \% \mathrm{p}$ & & $80(64-109)$ & ND \\
\hline Chest radio & raphic stages I/II/III & $5 / 11 / 3$ & Normal \\
\hline \multicolumn{4}{|c|}{$\begin{array}{l}\text { Data are presented as median (interquartile range), unless otherwise stated. } \\
\text { VC: vital capacity; } D \mathrm{~L}, \mathrm{CO} \text { : carbon monoxide diffusing capacity of the lung; ND: } \\
\text { not done. }{ }^{\#}: \mathrm{n}=19 ;{ }^{\top}: \mathrm{n}=20^{+}{ }^{+}: \text {median (minimum-maximum); }{ }^{\text {s: values are }} \\
\text { shown as per cent of predicted [16]. }\end{array}$} \\
\hline
\end{tabular}

\section{BAL procedure and handling of cells}

BAL was performed as previously described [13]. The BALF was strained through a Dacron net, the cells were centrifuged at $400 \times g$ for $10 \mathrm{~min}$ at $4^{\circ} \mathrm{C}$ and resuspended in RPMI 1640 medium (Gibco, Paisley, UK). BALF cells were counted and cell viability was determined (median 95\%) by trypan blue exclusion. Ficoll-Hypaque (Pharmacia, Uppsala, Sweden) gradient centrifugation was used to separate peripheral blood mononuclear cells. The separated cells were washed twice and diluted in RPMI 1640.

\section{Phenotypic analysis and flow cytometry}

BALF and PB cells from all patients and controls were incubated for $30 \mathrm{~min}$ at $4{ }^{\circ} \mathrm{C}$ with direct-labelled cytochrome (Cy5) anti-CD3 and phycoerythrin (PE) anti-CD56 (Becton Dickinson, Mountain View, CA, USA), washed twice and fixed in Cell-fix (Becton Dickinson, Stockholm, Sweden). For determination of KIR and CD94/NKG2A receptors, BALF and PB cells from a limited number of patients $(n=10)$ and controls $(n=7)$ were incubated with unlabelled DX9 (specific for p70 or KIR3DL1), DX22 (specific for CD94), DX27 (specific for p58 or KIR2DL2, 3 and KIR2DS2), DX31 (anti-KIR3DL2; antibodies were kindly provided by L. Lanier, DNAX Research Institute, Palo Alto, CA, USA) and NKG2A-specific (Z199, Immunotech, Marseille, France) monoclonal antibodies (Mabs) for $20 \mathrm{~min}$ at $4^{\circ} \mathrm{C}$ and washed twice. Fluorescein isothiocyanate (FITC)-conjugated F2 fragments of rabbit anti-mouse immunglobulin were added for the detection of bound primary antibodies. Normal mouse serum diluted 1:500 was used to block remaining rabbit anti-mouse immunoglobulin before adding the Cy5-conjugated CD3 and PE-conjugated CD56 Mabs. A combination of the three Mabs detecting KIR3DL1 (DX9), KIR2DL2, KIR2DL3, KIR2DS2 (DX27) and KIR3DL2 (DX31) that were used in the current study is referred to as KIRs. Each of these receptors was also studied separately in a limited number of patients ( $n=6$ (DX9), $n=6$ (DX27) and $n=9$ (DX31), respectively). Cells were analysed in a flow cytometer (FACScalibur; Becton Dickinson) within $24 \mathrm{~h}$. Lymphocytes were easily distinguished on the basis of forward and side scatters, and the percentage of the NK cells (CD3-CD56+) and CD56+ T-lymphocytes (CD3+CD56+) were scored by calculation of positive cells in each specific quadrant. Isotypematched negative control antibodies always stained $<1 \%$ cells. To distinguish between the CD56bright (high intensity) and CD56dim NK cell subpopulations, two regions were established within the CD3-CD56+ NK cells in accordance with previous reports $[14,15]$.

\section{Cell stimulation and intracellular staining}

BALF and blood cells from four patients were examined for cytokine production. A total of $2 \times 10^{6}$ cells from BALF and PB were resuspended in $1 \mathrm{~mL}$ of culture medium (RPMI 1640, supplemented with $5 \%$ foetal calf serum, $1 \%$ penicillin/ streptomycin and 1\% L-glutamine $2 \mathrm{mM}$ solution). Cells were stimulated by the addition of $10 \mathrm{ng} \cdot \mathrm{mL}^{-1}$ phorbol 12-myristate13-acetate (PMA; Sigma, Stockholm, Sweden) and $0.5 \mu \mathrm{M}$ ionomycin (Sigma) in the presence of the protein transport inhibitor brefeldin A $10 \mu \mathrm{g} \cdot \mathrm{mL}^{-1}$ (Sigma). After a 5-h incubation at $37^{\circ} \mathrm{C}$ and $5 \% \mathrm{CO}_{2}$, cells were harvested and stained for surface markers CD3 and CD56 for $20 \mathrm{~min}$ at $4^{\circ} \mathrm{C}$. After incubation, the cells were washed twice with PBS and then 
incubated with Cytofix/Cytoperm solution (Pharmingen, San Diego, CA, USA) for $20 \mathrm{~min}$ at $4^{\circ} \mathrm{C}$. Thereafter, cells were washed with Perm/Wash solution (Pharmingen), followed by intracellular staining with FITC-conjugated anti-IFN- $\gamma$ and anti-TNF- $\alpha$ (Pharmingen) for $30 \mathrm{~min}$ at $4^{\circ} \mathrm{C}$. Finally, cells were washed and fixed with Cellfix (Becton Dickinson), and the expression of cytokines was analysed by flow cytometry.

\section{Statistical analysis}

The nonparametric Wilcoxon matched-pair statistical test was used for calculation of the statistical significance of KIRs expression between paired samples of BALF and PB in patients and controls. For comparison between proportions of NK and CD56+ T-cells in BALF and PB in patients and controls, and also the expression of KIRs between patients and controls in each compartment, the Mann-Whitney U-test was used. Furthermore, for comparison between CD56bright and CD56dim NK cell subpopulations in BALF and PB from patients and controls, the Mann-Whitney U-test was used. All results are presented as median values, with interquartile values as the range in parentheses, unless otherwise stated. A p-value $<0.05$ was regarded as significant.
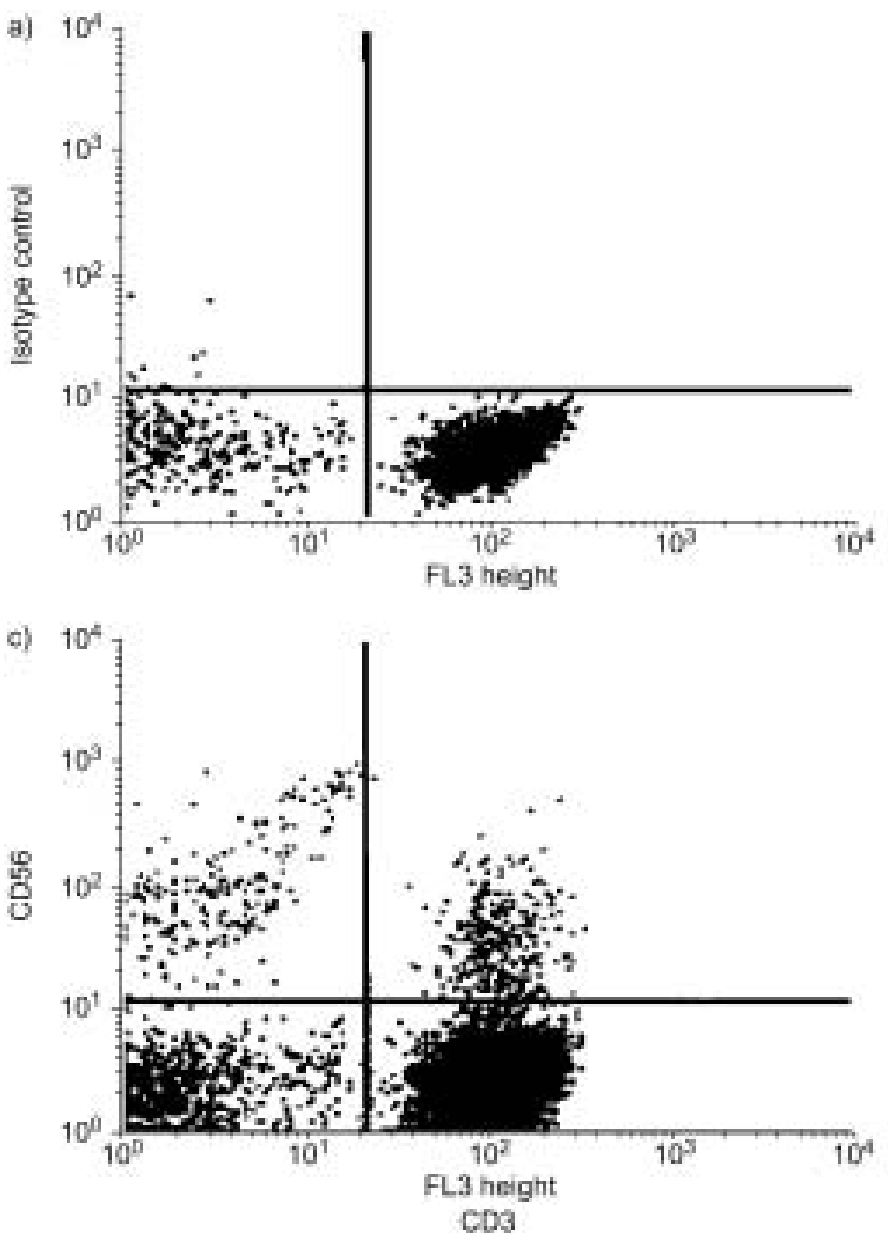

\section{RESULTS}

BALF and paired PB cells from a total of 19 sarcoidosis patients and 20 healthy controls were analysed in this study. Total and differential cell counts for BALF, and CD4/CD8 ratios are presented in table 1 .

\section{NK and CD56+ T-cell population in BALF and PB}

Flow cytometric analysis of CD3 and CD56 expression was used to identify NK cells (CD3-CD56+) and CD56+ T-cells, and to determine the frequency of these populations in BALF and PB in patients and controls. Representative dotplots from a sarcoidosis patient showing NK and CD56+ T-cell populations in BALF and PB are shown in figure 1. The relative frequency of NK cells in BALF lymphocytes (median 5.4\% (lower 3.6-upper 6.8 quartiles)) was significantly lower compared with PB (16.3\% (9.7-22.8)) in patients $(p<0.001)$. The same pattern was noted for the healthy controls' BALF (4.5\% (3.7-6.8)) and PB (13.1\% (10.0-19.3)) NK cells $(\mathrm{p}<0.001)$.

As shown in figure 2, there was no significant difference between the frequency of NK cells in BALF and PB between patients and controls. However, there were significantly more CD56+ T-
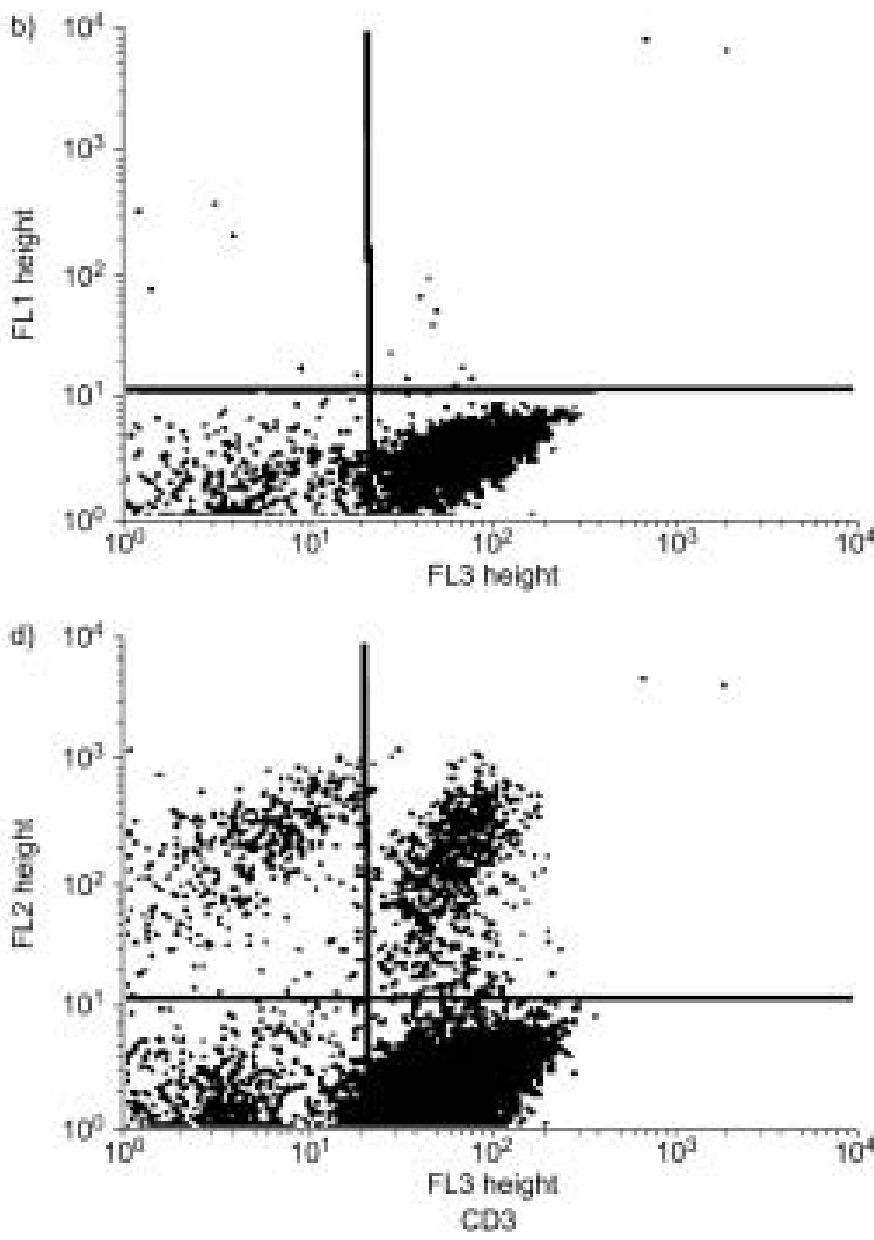

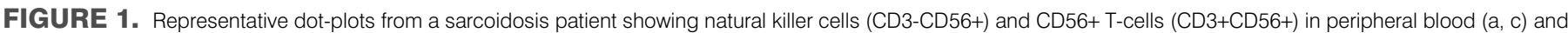

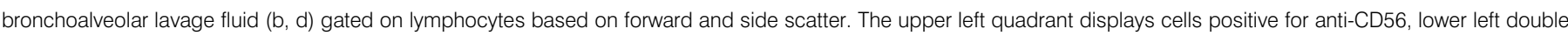
negative cells, upper right cells positive for both anti-CD56 and anti-CD3, and lower right cells positive for anti-CD3 monoclonal antibody. FL: fluorescence. 
cells in patients' $\mathrm{PB}(14.9 \%(6.2-31.9))$ when compared with healthy controls $(5.7 \%(3.7-7.0) ; \mathrm{p}<0.005)$, while the number of BALF CD56+ T-cells was not significantly different in patients (5.8\% (2.0-8.9)) and controls (3.6\% (2.3-5.8); $\mathrm{p}=\mathrm{NS}$; fig. 2).

\section{CD56bright and CD56dim NK cell subsets in BALF and PB}

When analysing the frequencies of the CD56bright and CD56dim NK cell subsets in patients, significantly more CD56bright NK cells were found in BALF (10.7\% of total NK cells (5.0-17.0)) when compared to $\mathrm{PB}(3.6 \%$ (2.5-6.1); $\mathrm{p}<0.0005)$. In contrast, in healthy controls, the number of CD56bright cells in BALF (2.1\% (0.9-4.5)) was at the same level as in PB (2.9\% (1.8-4.4); fig. 3). In accordance with these results, the level of the CD56dim subset in BALF (89.3\% (82.8-95.0)) was significantly reduced compared with PB CD56dim NK cells $(95.6 \%(93.9-96.7))$ in patients $(p<0.001)$, whereas, in controls, the number of CD56dim cells was similar in BALF (98.0\% (95.5-99.2)) and PB (96.5\% (94.4-97.9); $\mathrm{p}=\mathrm{NS})$.

There were significantly more CD56bright cells present in the BALF of patients than of controls $(p<0.0005)$, whereas no difference was found for PB CD56bright NK cells in patients and controls (fig. 3).

\section{Expression of KIRs on NK and CD56+ T-cell subsets}

The expression of KIRs and CD94/NKG2 receptors was evaluated on NK and CD56+ T-cells in the BALF and PB of 10 patients and seven healthy controls. In patients, there was a significant reduction in KIR-expressing NK cells in BALF when compared with $\mathrm{PB}$, and a similar pattern was observed for CD56+ T-cells (table 2; fig. 4). In contrast, the CD94 receptor was expressed by a significantly larger fraction of NK cells in BALF than in PB (table 2; fig. 4). In a limited number of samples from patients (not controls), these receptors were also investigated separately, as was the C-type lectin-like receptor NKG2A, i.e. one of the three NKG2 receptor molecules binding the CD94 molecule. When investigating each inhibitory receptor (p70, p58, KIR3DL2, CD94 and NKG2A) separately, in a subgroup of patients $(n=6), p 58$ in particular was found to be expressed by a reduced number of NK cells in BALF when compared with PB (10.6\% (8.1-16.9) and 22.5\% (19.5-24.6), respectively; $\mathrm{p}<0.05)$. The $\mathrm{C}$-type lectin-like receptor NKG2A is expressed together with CD94 as a heterodimeric molecule and, similar to CD94, it was expressed by a majority of NK and CD56+ T-cells in the BALF of patients when compared with PB (data not shown). The frequency of NK cells expressing KIRs and CD94 receptors in BALF was similar to PB in controls (table 2). In addition, the expression of KIRs and CD94 receptors on CD56+ T-cells in BALF compared with PB from controls showed no statistically significant difference (table 2). However, there was a strong tendency towards lower numbers of KIR-expressing NK cells in both BALF and PB of patients compared with healthy controls $(p=0.09$; table 2$)$. The number of CD94-expressing NK and CD56+ T-cells in BALF and PB was heterogenous and not different from patients.
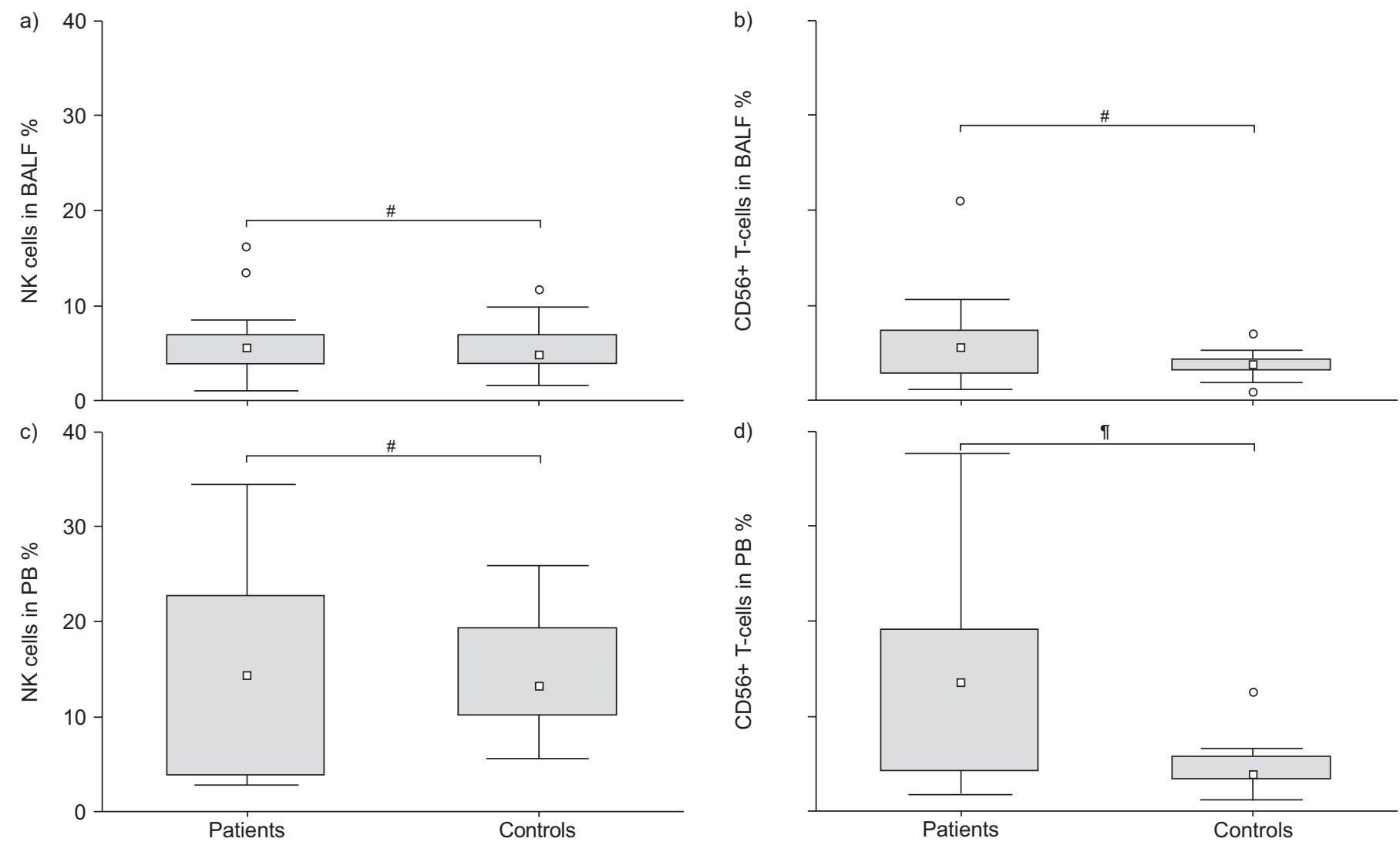

FIGURE 2. Percentage of natural killer (NK) cells and CD56+ T-cells in bronchoalveolar lavage fluid (BALF) and peripheral blood (PB) from patients and healthy controls The box represents the interquartile values (25-75\%) and the whiskers represent the non-outlying maximum and minimum. $\bigcirc:$ outliers; $\square:$ median. ${ }^{\#}: p=$ NS; ${ }^{\top}: p<0.005$. 

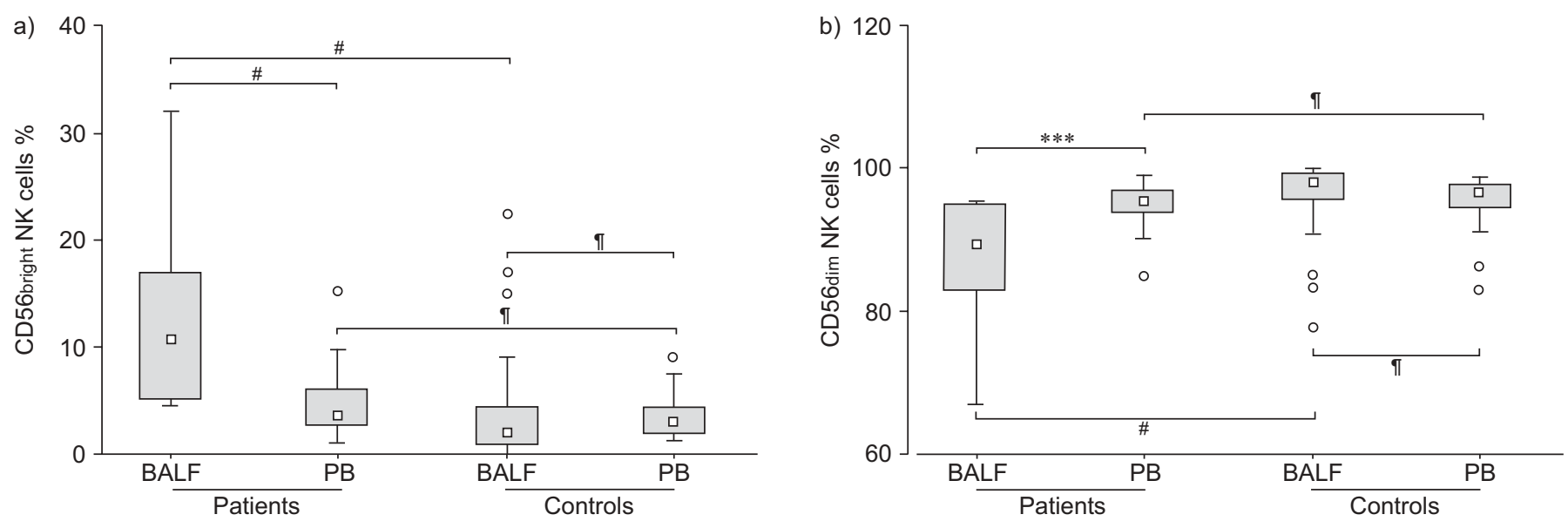

FIGURE 3. Frequencies of natural killer (NK) cell subsets (CD56bright (a) and CD56dim (b)) in bronchoalveolar lavage fluid (BALF) and peripheral blood (PB) from patients and controls. The box represents the interquartile values (25-75\%) and the whiskers represent the non-outlying maximum and minimum. $\bigcirc$ : outliers; $\square$ : median. $* * *$ : $p<0.001 ;{ }^{*}: p<0.0005 ;{ }^{\bullet}: p=N S$.

TABLE 2 Percentage of BALF and PB NK cells and CD56+ T-cells expressing KIRs and CD94 receptors in patient and controls

\begin{tabular}{llllllll} 
& Receptors & BALF patients & p-value & PB patients & BALF controls & p-value & PB controls \\
\hline \multirow{2}{*}{ NK cells } & KIRs & $15.4(5.3-21.9)^{\#}$ & $<0.01$ & $40.9(34.9-49.4)^{\bullet}$ & $31.5(15.6-44.9)^{\#}$ & NS & $49.8(45.0-60.5)^{\bullet}$ \\
& CD94 & $81.8(75.8-91.9)$ & $<0.05$ & $64.8(39.9-86.5)$ & $83.1(80.2-89.9)$ & NS & $73.9(66.2-87.7)$ \\
CD56+ T-cells & KIRs & $15.0(8.6-20.3)$ & $<0.05$ & $26.8(17.7-39.0)$ & $23.5(16.8-30.4)$ & NS & $32.8(7.9-37.5)$ \\
& CD94 & $58.9(38.1-74.7)$ & NS & $58.2(26.4-69.2)$ & $54.5(45.9-71.9)$ & NS & $51.2(45.4-64.5)$ \\
& & & & &
\end{tabular}

Data are presented as median (interquartile range), unless otherwise stated. BALF: bronchoalveolar lavage fluid; PB: peripheral blood; NK: natural killer; KIR: killer cell immunoglobulin-like receptor. ${ }^{\#}: p=0.09$ for comparing KIRs expression in BALF between patients and controls; ${ }^{\natural}: p=0.09$ for comparing KIRs expression in PB between patients and controls; NS: nonsignificant.

\section{Cytokine production by NK and CD56+ T-cells}

Following stimulation with PMA and ionomycin, cytokine production by NK cells and CD56+ T-cells was determined by three-colour flow cytometry in four patients. An increased proportion of NK cells produced IFN- $\gamma$ in BALF (55.4\% (13.8$70.4))$, as compared with PB (21.4\% (3.1-51.3)). Similarly, the fraction of TNF- $\alpha$-producing NK cells was increased in BALF (42.1\% (21.9-57.1)), as compared with PB (0.3\% (0.3-18.5)). In addition, CD56+ T-cells produced more IFN- $\gamma(66.3 \%$ (55.677.1)) and TNF- $\alpha$ (78.6\% (43.9-81.4)) in BALF compared with PB (28.9\% (6.7-41.1) and 5.2\% (4.9-43.3), respectively). Dotplots from a representative patient flow cytometry staining are shown in figure 5 .

\section{DISCUSSION}

In this study, two distinct lymphocyte populations, i.e. NK (CD3-CD56+) and CD56+ T-cells, were characterised in the BALF and PB of patients with sarcoidosis and healthy individuals. The frequencies of these subsets, their phenotype with respect to their expression of KIRs and C-type lectin (CD94/NKG2A) receptors, as well as the distribution of CD56bright and CD56dim NK cell subsets, were determined. Furthermore, the cytokine-producing capacity of NK cells and CD56+ T-cells in the BALF and PB of patients was studied. To the current authors' knowledge, this is the first study in which such an experimental approach has been used to study lung and blood NK cell subsets and CD56+ T-cell populations in sarcoidosis.

In line with other reports of patients with sarcoidosis as well as healthy controls, the current study found a relatively low percentage of NK cells in BALF when compared with PB $[8,9$, 17]. However, especially in patients, NK cells in BALF were phenotypically distinct compared with NK cells in $\mathrm{PB}$, with a minority expressing KIRs and a majority expressing the inhibitory CD94/NKG2A receptor. In contrast to previous studies, this study found no excess of NK cells in the PB of patients $[8,9]$.

NK cells can act without prior sensitisation to produce cytokines, predominantly IFN- $\gamma$, and may cause cytolysis of target cells [18]. Human NK cell reactivity is controlled by inhibitory receptors specific for MHC class I, including KIRs and CD94/NKG2A receptors. These receptors were first described on NK cells and, subsequently, on subsets of Tlymphocytes. They inhibit NK cells from killing target cells with normal MHC class I expression, but enable the killing of cells with a deviant MHC class I expression [19]. Defective KIR expression and/or the absence of MHC class I complexes on the surface of normal cells may be of importance in autoimmune diseases [20]. Expression of KIRs and CD94 

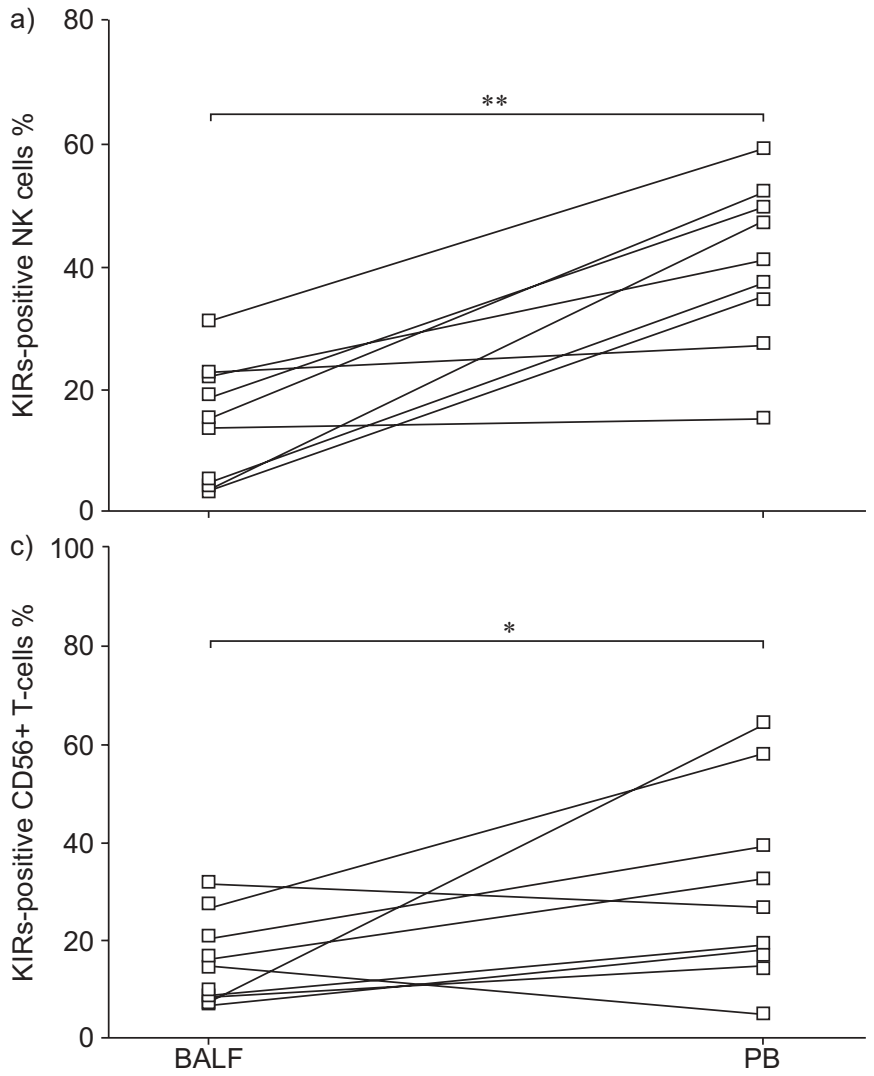
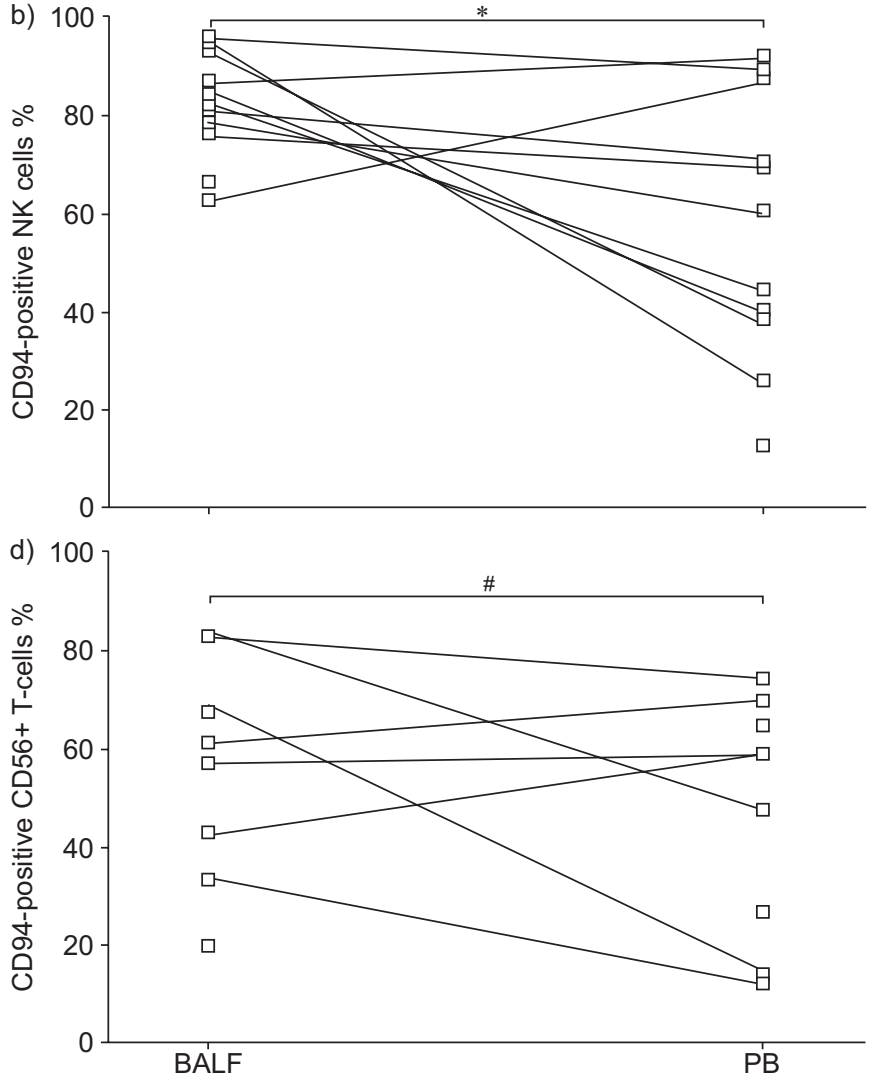

FIGURE 4. Expression of killer cell immunoglobulin-like receptors (KIRs) and CD94 receptors on natural killer (NK; a, b) and CD56+ T-cell subsets (c, d) in bronchoalveolar lavage fluid (BALF) and peripheral blood (PB) from patients. The number of patients in each experiment varied (NK cells: KIRs $n=9$, CD94 $n=10$; and CD56+ T-cells: KIRs $n=9$, CD94 $n=7$ ). *: $p<0.05 ;{ }^{* *}: p<0.01 ; *: p=N S$.

receptors on T-cell subsets has previously been studied by the current authors' group, showing elevated numbers of CD8+CD94+ T-cells in BALF and a reduction of KIR+CD8+ BALF T-cells compared with $\mathrm{PB}$, i.e. a pattern similar to the one described in the present study for NK cells [21].

The enrichment of the CD94high KIRlow NK subpopulation within the lung could reflect a preferential recruitment of this specific subset from the periphery, and these NK cells may contribute to the local ongoing immune response by secreting T-helper (Th)1 cytokines, which exaggerate the inflammatory response in the lower respiratory tract of these patients.

AgOSTINI et al. [8] have previously demonstrated an increased in vitro cytotoxic activity by NK cells in the $\mathrm{PB}$ of patients with active sarcoidosis, whereas, in contrast, the small proportion of NK cells found in BALF was almost inactive. NK cell function is, however, not restricted to the cytotoxicity of tumour or virally infected cells. By secreting cytokines, such as IFN- $\gamma$, NK cells can also contribute towards the establishment of an inflammatory, Th1-oriented response [18]. For example, in rheumatoid arthritis, the synovial fluid NK cell population has an enhanced capacity to secrete IFN- $\gamma$ in response to interleukin-12 and -15 when compared with blood NK cells [22]. In the current study, an assay that could detect cytokine secretion at a single-cell level by flow cytometry was used, and the analysis of cytokine production by BALF NK cells revealed a significant capacity of NK cells to produce IFN- $\gamma$ and TNF- $\alpha$. Even though T-cells are considered to have a major role in the pathogenesis of sarcoidosis, it was hypothesised that NK cells and CD56+ T-cells also contribute by, for example, activating macrophages through their cytokine production.

NK cells can be divided into two subsets according to CD56 surface-density expression, i.e. CD56bright and CD56dim [23]. CD56bright NK cells normally represent a relatively small proportion of NK cells and they have been reported to be more immunoregulatory, principally through cytokine production. The CD56bright NK cells express reduced levels of KIRs and increased levels of CD94/NKG2A, which are characteristic features of this subpopulation. Conversely, CD56dim cells express high levels of KIRs but lower levels of CD94 receptors, and early functional studies have shown that the CD56dim subset is more cytotoxic [1]. In the present study, the frequency of CD56bright and CD56dim cells in the BALF and PB of patients and healthy volunteers was investigated. It was observed that, in patients, the frequency of CD56bright NK cells in BALF was significantly higher compared with $\mathrm{PB}$, and also that lung NK cells are more likely to express CD94, and less likely to express KIRs, than PB NK cells. This supports the current hypothesis that the accumulated NK cells in the lungs of the patients belong to a distinct NK cell subpopulation, i.e. CD56bright CD94highKIRlow, with the capacity to produce large amounts of 

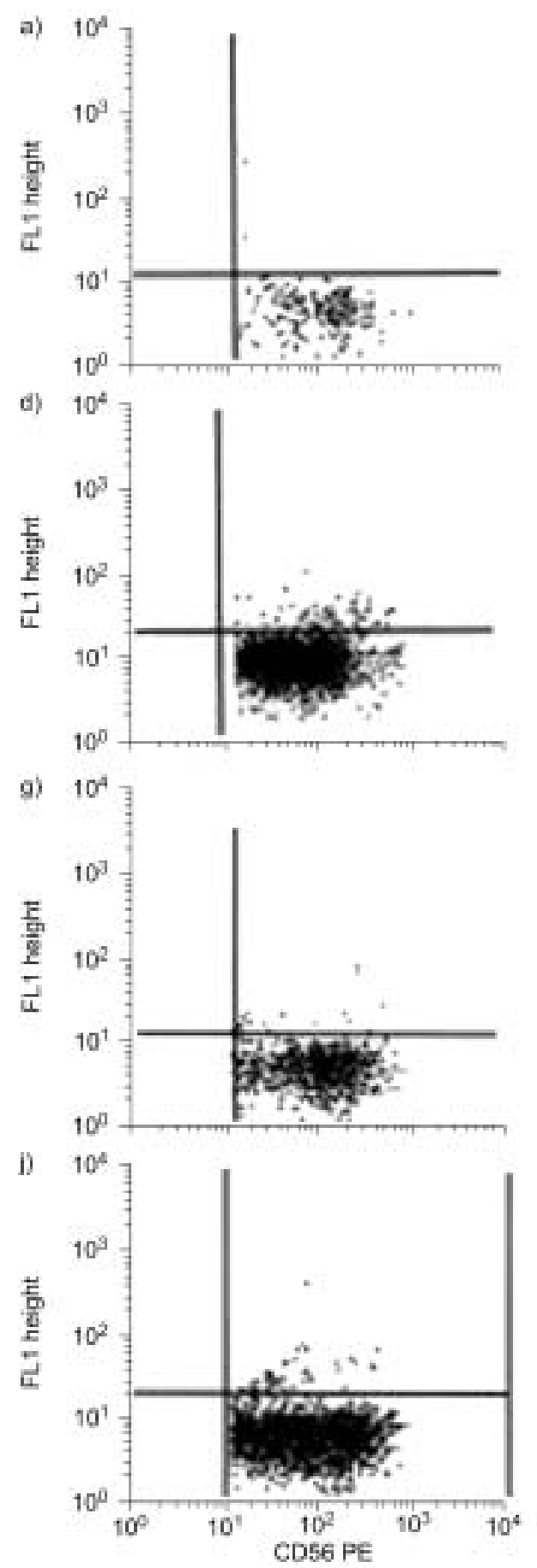

b)

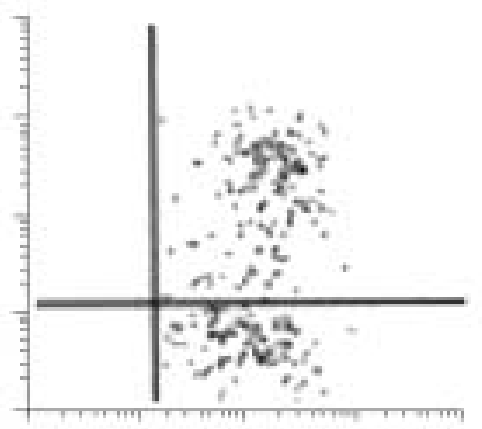

ө)

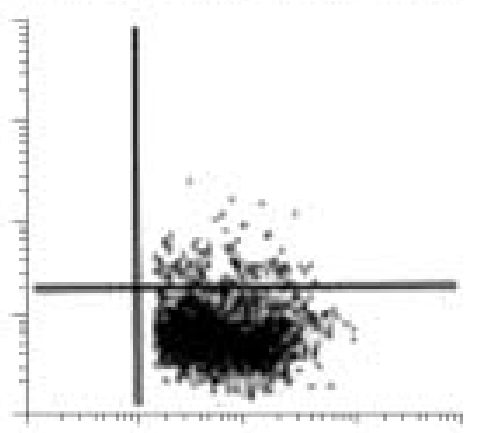

h)

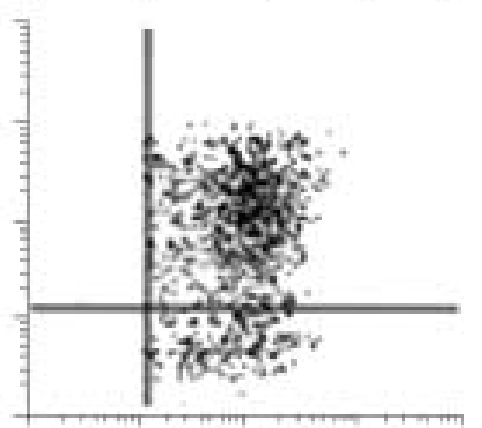

k)

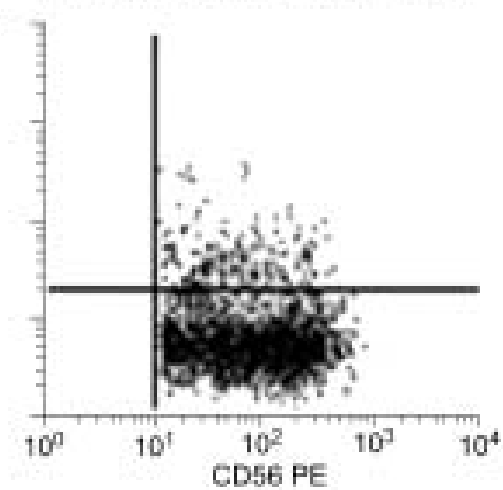

c)

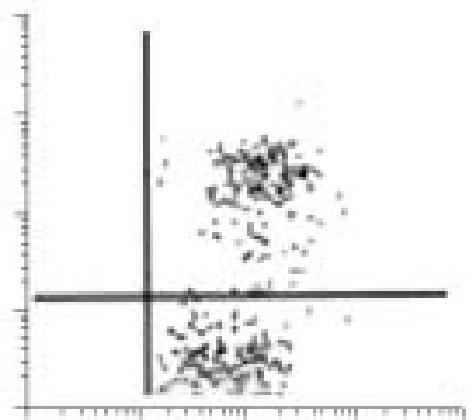

f)

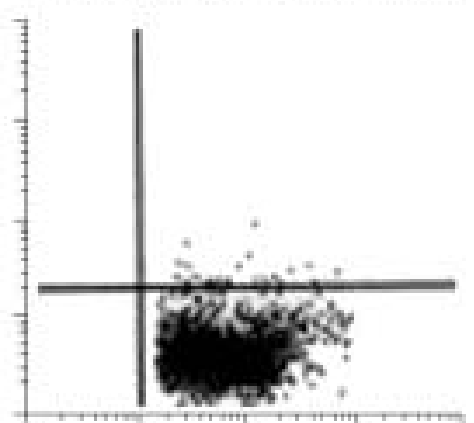

i)

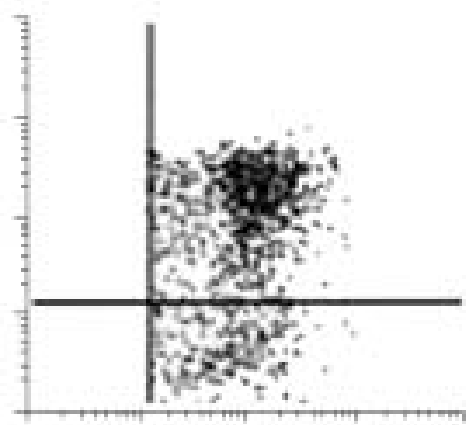

1)

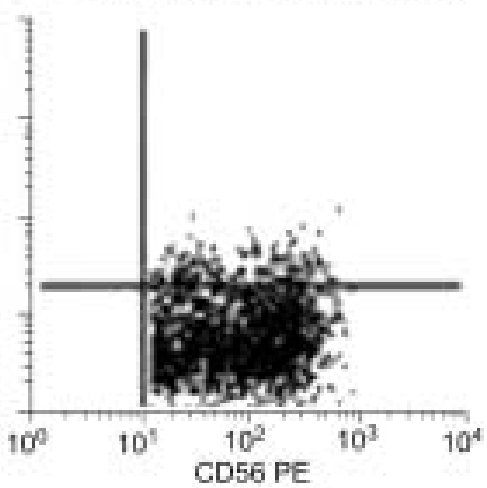

FIGURE 5. Flow cytometry dot-plots of bronchoalveolar lavage fluid $(a-c, g-i)$ and peripheral blood $(d-f, j-l)$ natural killer (NK; a-f) cells and CD56+ T-cells ( $g-l)$ from a representative patient. Cells were stained with fluorescein isothiocyanate-labelled antibodies against isotype control (a, $d, g, j)$, interferon- $\gamma(b, e, h, k)$ and tumour necrosis factor- $\alpha(\mathrm{c}, \mathrm{f}, \mathrm{i}, \mathrm{l})$ and phycoerythrin-labelled anti-CD56. NK cells are gated on CD3-CD56+ cells and CD56+ T-cells are gated on CD3+CD56+ cells. FL: fluorescence; PE: phycoerythrin.

cytokines such as IFN- $\gamma$ and TNF- $\alpha$, but to have less cytotoxic activity. In fact, the CD56bright NK cell population was significantly larger in the BALF of patients compared with the BALF of controls, further implicating the presence of these cells in the sarcoid inflammatory process. It has been shown that CD56bright NK cells are enriched at inflammatory sites and can interact with other mononuclear cells to promote the inflammatory process. In line with this finding, there was also a more pronounced lung accumulation of CD94high and KIRlow NK cells in the patient group when compared with controls.

CD56+ T-cells are highly cytolytic and can display non-MHCrestricted cytotoxicity $[5,6]$. Most of these CD56+ T-cells 
express CD8 and appear more efficient in IFN- $\gamma$ production, as compared with CD56- T-cells [7]. However, the functional roles of CD56+ T-cells, apart from their cytotoxic capacity, are not well defined yet, but they may have particular importance in immunoregulation. They have been found in high frequencies in the liver [24].

Significantly elevated numbers of CD56+ T-cells were found in PB from patients when compared with controls, and a selection of KIR-expressing CD56+ T-cells in BALF. There were also more IFN- $\gamma$ - and TNF- $\alpha$-producing CD56+ T-cells in BALF compared with PB. The full relevance of these findings is still speculative; nevertheless, the increased number of CD56+ Tcells in the PB of patients may suggest that the CD56+ T-cell subset can play an important role in regulating the immune response in sarcoidosis, although further analyses, such as investigating other cytokines, are required to understand the role of this lymphocyte subset in the disease. Sarcoidosis is a systemic disorder and an elevated level of serum inflammatory mediators indicates the systemic nature of this disease.

In conclusion, in this study, the natural killer and CD56+ T-cell subsets in sarcoidosis have been characterised, a distinct phenotype was found at the site of inflammation, i.e. the lungs, and, furthermore, the natural killer cell subsets differed significantly from the bronchoalveolar lavage fluid natural killer cells of healthy controls. The particular subset of natural killer cells found to accumulate in the lungs has the capacity to produce large amounts of interferon- $\gamma$ and tumour necrosis factor- $\alpha$, and may thereby enhance the inflammatory activity in the lungs. In addition, significantly elevated levels of CD56+ Tcells in the blood of patients were observed. Further experiments are essential to determine the function of CD56+ T-cells in the blood of patients. Characterising these cells may lead to a better understanding of the mechanisms behind the inflammatory process in sarcoidosis.

\section{ACKNOWLEDGEMENTS}

The authors would like to thank L. Lanier (DNAX Research Institute of Molecular and Cellular Biology, Palo Alto, CA, USA), for generously providing the KIR-specific monoclonal antibodies. They would also like to thank L. Berg and C. Teixeira de Matos for helpful discussions and methodological suggestions, and S. Gripenbäck for providing the healthy control material.

\section{REFERENCES}

1 Cooper MA, Fehniger TA, Caligiuri MA. The biology of human natural killer-cell subsets. Trends Immunol 2001; 22: 633-640.

2 Carson WE, Fehniger TA, Caligiuri MA. CD56bright natural killer cell subsets: characterization of distinct functional responses to interleukin-2 and the c-kit ligand. Eur J Immunol 1997; 27: 354-360.

3 Gottschalk LR, Bray RA, Kaizer H, Gebel HM. Two populations of CD56 (Leu-19)+/CD16+ cells in bone marrow transplant recipients. Bone Marrow Transplant 1990; 5: 259-264.

4 Middleton D, Curran M, Maxwell L. Natural killer cells and their receptors. Transpl Immunol 2002; 10: 147-164.
5 Lanier LL, Le AM, Civin CI, Loken MR, Phillips JH. The relationship of CD16 (Leu-11) and Leu-19 (NKH-1) antigen expression on human peripheral blood NK cells and cytotoxic T lymphocytes. J Immunol 1986; 136: 44804486.

6 Pittet MJ, Speiser DE, Valmori D, Cerottini JC, Romero P. Cutting edge: cytolytic effector function in human circulating CD8+ T cells closely correlates with CD56 surface expression. J Immunol 2000; 164: 1148-1152.

7 Ohkawa T, Seki S, Dobashi H, et al. Systematic characterization of human CD8+ T cells with natural killer cell markers in comparison with natural killer cells and normal CD8+ T cells. Immunology 2001; 103: 281-290.

8 Agostini C, Trentin L, Zambello R, et al. Phenotypical and functional analysis of natural killer cells in sarcoidosis. Clin Immunol Immunopathol 1985; 37: 262-275.

9 Semenzato G, Agostini C, Pezzutto A, et al. Distribution of natural killer cells in sarcoidosis. J Clin Lab Immunol 1984; 13: 25-28.

10 Robinson BW, Pinkston P, Crystal RG. Natural killer cells are present in the normal human lung but are functionally impotent. J Clin Invest 1984; 74: 942-950.

11 Consensus conference: activity of sarcoidosis. Third WASOG meeting, Los Angeles, USA, September 8-11, 1993. Eur Respir J 1994; 7: 624-627.

12 Costabel U. CD4/CD8 ratios in bronchoalveolar lavage fluid: of value for diagnosing sarcoidosis? Eur Respir J 1997; 10: 2699-2700.

13 Eklund A, Blaschke E. Relationship between changed alveolar-capillary permeability and angiotensin converting enzyme activity in serum in sarcoidosis. Thorax 1986; 41: 629-634.

14 Lima M, Teixeira MA, Queiros ML, et al. Immunophenotypic characterization of normal blood CD56+lo versus CD56+hi NK-cell subsets and its impact on the understanding of their tissue distribution and functional properties. Blood Cells Mol Dis 2001; 27: 731-743.

15 Tarazona R, Casado JG, Delarosa O, et al. Selective depletion of CD56(dim) NK cell subsets and maintenance of CD56(bright) NK cells in treatment-naive HIV-1-seropositive individuals. J Clin Immunol 2002; 22: 176-183.

16 Quanjer PH, Tammeling GJ, Cotes JE, Pedersen OF, Peslin R, Yernault JC. Lung volumes and forced ventilatory flows. Report working party standardization of lung function tests, European Community for Steel and Coal. Official Statement of the European Respiratory Society. Eur Respir J 1993; 6: Suppl. 16, S5-S40.

17 Meyer KC, Soergel P. Variation of bronchoalveolar lymphocyte phenotypes with age in the physiologically normal human lung. Thorax 1999; 54: 697-700.

18 Lanier LL. The origin and functions of natural killer cells. Clin Immunol 2000; 95: S14-S18.

19 Lanier LL. Natural killer cell receptors and MHC class I interactions. Curr Opin Immunol 1997; 9: 126-131.

20 Fu Y, Nathan DM, Li F, Li X, Faustman DL. Defective major histocompatibility complex class I expression on lymphoid cells in autoimmunity. J Clin Invest 1993; 91: 2301-2307.

21 Mizuki M, Eklund A, Grunewald J. Altered expression of natural killer cell inhibitory receptors (KIRs) on $\mathrm{T}$ cells in 
bronchoalveolar lavage fluid and peripheral blood of sarcoidosis patients. Sarcoidosis Vasc Diffuse Lung Dis 2000; 17: 54-59.

22 Dalbeth N, Callan MF. A subset of natural killer cells is greatly expanded within inflamed joints. Arthritis Rheum 2002; 46: 1763-1772.

23 Cooper MA, Fehniger TA, Turner SC, et al. Human natural killer cells: a unique innate immunoregulatory role for the CD56(bright) subset. Blood 2001; 97: 31463151.

24 Norris S, Doherty DG, Collins C, et al. Natural T cells in the human liver: cytotoxic lymphocytes with dual $\mathrm{T}$ cell and natural killer cell phenotype and function are phenotypically heterogenous and include Valpha24-JalphaQ and gammadelta $\mathrm{T}$ cell receptor bearing cells. Hum Immunol 1999; 60: 20-31. 